NOTICIAS Y COMENTARIOS 


\title{
DESARROLLO LOCAL, GEOGRAFÍA Y ANÁLISIS TERRITORIAL INTEGRADO: ALGUNOS EJEMPLOS APLICADOS
}

\author{
Enrique A. Moltó Mantero \\ María Hernández Hernández \\ Departamento de Análisis Geográfico Regional \\ Universidad de Alicante
}

\section{RESUMEN}

Las transformaciones registradas en la situación económica, social y política actual se traducen en una reorganización del territorio a todas las escalas. Frente a este proceso de globalización, se ha generado una notable expansión de los estudios relacionados con la escala local. El territorio, entendido como el campo de relaciones e interdependencias entre factores diversos, es uno de los elementos que refuerzan y consolidan la función y el significado de la Geografía. El análisis de la estructura ecológico-ambiental y socioeconómica es fundamental en toda actuación vinculada al desarrollo. En estos análisis debería predominar el carácter integrado, no obstante, no son extraños aquéllos donde priman los enfoques sectoriales o los que resultan de la mera suma de elementos individualizados.

Palabras clave: desarrollo local, globalización, análisis territorial, territorio, Geografía.

\section{ABSTRACT}

The changes produced in the current economic, social and political position turn into a land reorganization at every scale. Facing this world-wide extension process, it is generated a remarkable enlargement of researches that have to do with local scale. The land, understood as the area of relationships and interdependences among different factors, is one of the elements that strengthen and consolidate Geography's significance and function. The analysis of ecological and socio-economic structure is essential in every proceeding linked to development. In these studies, ought to prevail the integrated condition, nevertheless, studies where approaches relating to a particular sector or ones resulted of a simple addition of elements are very common.

Key words: local development, world-wide whole process, land analysis, land, Geography. 


\section{Introducción}

El interés por el estudio de los procesos de desarrollo en el más amplio sentido de la palabra y a distintas escalas ha ido en aumento en las últimas décadas. Esta difusión ha ido asociada a la «apropiación» que de él han realizado profesionales vinculados a las ciencias económicas. La divulgación del concepto de desarrollo local, entendido como una noción que engloba conceptos no estrictamente económicos, es más reciente; no obstante, si se considera como un objetivo perseguido por cualquier sociedad, aunque no reciba tal denominación, cabe remontarlo a la génesis de las primeras civilizaciones.

Para comprender en su totalidad la compleja noción de «desarrollo local» hemos de incidir en la evolución histórica del concepto «desarrollo» en sentido amplio, especialmente el registrado en los países occidentales. En una primera etapa, que coincide grosso modo con la primera revolución industrial, no se emplea el término desarrollo, sino crecimiento económico. El concepto de desarrollo entendido como sinónimo del de crecimiento económico no se utilizará hasta 1911, manteniéndose dicha equivalencia hasta la Segunda Guerra Mundial. Finalizado el conflicto bélico y como consecuencia de la difusión de principios que abogan por la necesidad de alcanzar ciertos niveles de equidad social, se incorporan nuevos planteamientos de desarrollo social a los estrictamente económicos, que se relacionan con la igualdad de género y la universalización de la cultura y del ocio (Calatrava, 1981). Estos planteamientos se generalizan a partir de la década de los setenta como consecuencia de las transformaciones en los procesos productivos. En la Declaración de la ONU de 1986 «Sobre el derecho al desarrollo» se describe este concepto como algo global, comprendiendo aspectos económicos, sociales, culturales y políticos. En ella se recoge igualmente que el fin de todo desarrollo debe ser la mejora constante del bienestar de los individuos y de la población en su conjunto. Esta declaración conlleva un fracaso de las teorías del desarrollo exclusivamente economicistas (Tortosa, 1997).

A finales de la década de los ochenta, se inicia una nueva fase en la evolución del concepto de desarrollo. Se considera insuficiente que éste sea intrageneracional, propugnándose la necesidad de que sea equitativo con las generaciones futuras. A los significados inherentes al término desarrollo se le incorpora la noción de sustentabilidad y, asociada a ésta, la del desarrollo sostenible. El concepto de la sustentabilidad, vinculado esencialmente a la preservación de valores ambientales, se centra en asegurar a las generaciones futuras un nivel de desarrollo similar al nuestro; sin embargo, un problema en numerosas ocasiones no considerado es el de desconocer cuál será la noción de bienestar en décadas venideras. La solución más factible a ese dilema es mantener nuestro stock de capital natural y transmitirlo para que nuestros descendientes puedan aplicar su concepto de bienestar. No obstante, deben ser consideradas igualmente las consecuencias derivadas de una posible aplicación de la noción de sustentabilidad en sentido estricto. Ésta puede implicar una seria limitación a nuestro propio desarrollo actual. Ello ha determinado que algunas propuestas insistan en la idea de una sustentabilidad en sentido débil en la que, junto al mantenimiento en lo posible del capital natural, se acepte también el traspaso de algunas transformaciones humanas (Calatrava, 1995), indispensables en algunos casos para la preservación de ciertos paisajes presuntamente «naturales» y en realidad de origen antropogénico (agrosistemas como las dehesas o los aterrazamientos característicos de la montaña media mediterránea, aprovechamientos de áreas húmedas como reservorio de caudales hídricos, explotaciones salineras, etc.). Esta última idea de desarrollo sostenible en sentido débil podría ser especialmente válida para determinadas concepciones geográ- 
ficas del territorio vinculadas al mantenimiento de parajes de alto valor paisajísticoambiental.

La evolución del concepto desarrollo no sólo ha ido ligada a los objetivos teóricos esbozados (económicos, sociales, de género, culturales, ambientales, etc.) sino que ha afectado también a su ámbito de aplicación. Si en los primeros momentos del desarrollo humano este sólo podía ser local como consecuencia del aislamiento relativo de las comunidades primitivas, las sociedades más evolucionadas fueron buscando desarrollos más globales, aunque siempre alrededor de la hegemonía de una o varias potencias. En el siglo pasado, en especial tras la Segunda Guerra Mundial, la internacionalización, sobre todo de la economía, aunque también de algunos valores sociales y culturales, nos conduce a una concepción global del desarrollo, contemplada ésta siempre desde los criterios de los países occidentales. En los últimos años, podemos hablar de una reacción en algunos ámbitos a ese desarrollo presuntamente global, un movimiento que defiende que la única opción viable para alcanzar íntegramente los valores asociados a un desarrollo integral reside precisamente en lo local, de forma tal que la suma de los diversos desarrollos locales, cada uno con su particular noción de bienestar, desemboque en un desarrollo global real (Benko, 1990).

La situación económica, social y política actual caracterizada por los cambios radicales en las técnicas y en los procesos productivos (avances tecnológicos, internacionalización de los circuitos del capital, la producción flexible y especializada, el postfordismo, etc.) conllevan una reorganización del territorio (Audiger, 1992; Brunet, 1995) a todas las escalas que se refleja en nuevas pautas de localización y de desarrollo regional, en la polarización del espacio o en la creación de nuevas relaciones socio-espaciales (Jameson, 1996; Benko y Struhmayer, 1997). La globalización, entendida como la introducción del sistema mundo en todos los lugares y en todos los individuos, constituye el estadio supremo de la internalización. La instantaneidad de la información gracias a la difusión de las nuevas tecnologías, la globalización de la economía, etc. generan una nueva concepción del espacio (Massey y Jess, 1995; Santos, 2000).

Estas cuestiones se traducen en una revalorización de los estudios relacionados con esos «nuevos lugares» (García Ballesteros, 2000) a los que se asocian conceptos como los de ciberespacio o aldea global. Paralelamente a este proceso de internalización socio-económica, se asiste a una valorización de aquellos aspectos que se vinculan a lo «local» (Di Meo, 1991; Cox, 1997). Este segundo hecho, que parece cuanto menos contradictorio con el de globalización, se relaciona con la revalorización de la denominada Nueva Geografía Regional (Nogué i Font, 1989) y las corrientes humanistas, que apoyadas en corrientes filosóficas del existencialismo y la fenomenología, convierten el espacio en lugar (Di Meo, 1998) al propugnar como uno de sus objetivos la comprensión de los hechos, el estudio de la experiencia humana directa en el lugar, entendido como espacio concreto, analizando sus significados, sus valores e intuiciones (Boira, 1995). Se vincula igualmente, y desde una óptica aplicada, a las políticas de desarrollo regional derivadas del Informe Brundtland, que abogan por un nuevo modelo de desarrollo económico basado en la promoción, entre otros, de los recursos endógenos.

Ambos procesos, globalización y revalorización de lo local, constituyen escalas de análisis de un mundo plural, cuya imbricación puede proporcionar análisis de gran valía desde el punto de vista del análisis territorial (Bailly, Guesnier, Paelick y Sallez, 1987; Cano,1990). No obstante, son frecuentes los trabajos que propugnan el empleo de una única escala de análisis, obviando que el estudio escalar es un elemento fundamental en el análisis territorial y uno de los rasgos que definen a la ciencia geográfica. En estas investigacio- 
nes se propugna el análisis de fenómenos globales porque permiten explicar tendencias generales, obviando escalas de trabajo más detalladas al tildarlas de «localistas» y vinculadas a la escuela regional clásica frente a concepciones «más modernas» y relacionadas con otras disciplinas más valoradas socialmente, aunque en ocasiones no constituyen más que meras abstracciones con escasa aplicabilidad en el mundo real. Es necesario, por tanto, conciliar ambas escalas de trabajo, pero también conceder su justo valor a cada tipo de análisis. La escala local, bien entendida, puede proporcionar resultados a partir de los cuales realizar abstracciones. Igualmente, estos análisis generalistas implican la supresión del trabajo de campo, de la comprobación de ciertas afirmaciones en un ámbito real; su utilización evitaría la especulación, la que podríamos denominar como «investigación de laboratorio».

\section{El territorio, objeto de estudio de la Geografía ¿punto de partida del desarrollo local?}

El análisis de las relaciones entre el hombre y el medio, eje de la investigación geográfica durante los dos últimos siglos y principal fundamento de la unidad de la disciplina en un mundo cada vez más encaminado a la especialización, otorga a la Geografía un lugar bastante bien definido entre las ciencias sociales. El territorio, entendido como el campo de relaciones e interdependencias entre factores diversos (Troitiño, 2000) es uno de los elementos que refuerzan y consolidan el papel y la identidad de la Geografía.

A pesar de esta relevancia, el proceso de mundialización de las relaciones sociales, económicas y de la información ha conducido a diversos autores a referirse a un nuevo concepto, el de la desterritorialización (Raffestain, 1984; Tuathail, 1998), entendido como la muerte del territorio al generarse un mundo sin naciones atravesado por flujos transnacionales e incluso algunos hablan de los «no lugares» (Auge, 1993), de mundos sin naciones como consecuencia del proceso de mundialización de las relaciones sociales, económicas y de la información que caracteriza a la sociedad actual. En realidad, esa creciente movilidad de los hombres, de los objetos y de las ideas gracias a las posibilidades derivadas de las nuevas comunicaciones nos situarían ante nuevas regiones (Boisier, 1994) y nuevas relaciones territoriales (Jordá, 1995; Estébanez, 1995).

El territorio se constituye en el elemento vertebrador de todos aquellos procesos que tienen incidencia sobre un cierto espacio en el que actúa una determinada sociedad. Consiguientemente, el análisis de la estructura territorial, de sus componentes y de su funcionamiento es uno de los elementos fundamentales en toda actuación vinculada al desarrollo local y fundamentada en la revalorización de su potencial endógeno (Esparcia, 1999). Estos estudios se caracterizan por una serie de cuestiones claves. Una de ellas es el carácter integrado de toda actuación. Siendo el análisis de la diferenciación del espacio en la superficie terrestre, de las relaciones espaciales de los fenómenos geográficos y de su organización en el espacio, otras cuestiones primordiales en estas investigaciones.

El carácter integrado de toda actuación sobre un determinado territorio es un concepto que requiere una mínima reflexión. Éste es asumido y aceptado por todos aquellos profesionales cuya labor se vincula a esta cuestión, dada la creciente demanda de proyectos relacionados con el desarrollo local, el medio ambiente, etc. La ordenación, por ejemplo, se ha convertido en uno de los más «novedosos y lucrativos inventos» en los últimos años, de ahí que numerosas disciplinas estén legitimadas para opinar sobre el territorio, sus procesos de planificación, su estructura u organización. Esta «invasión» ha sido posible en gran medida por el modelo de planificación territorial adoptada, generalmente sectorial. En el planea- 
miento urbanístico, por ejemplo, predominan las memorias informativas que reproducen literalmente variables biogeográficas, climáticas, hidrológicas, demográficas, etc. obtenidas de fuentes estadísticas. Sin embargo, no todos los expertos son capaces de desarrollar una visión integral del territorio. Uno de los principios esgrimidos para defender el carácter aplicado de la Geografía ha sido la superioridad de los geógrafos, por su formación, sobre otros profesionales a la hora de gestionar el territorio. Las oportunidades del geógrafo en el campo profesional dependen, sobre todo, de la habilidad que éste demuestre para manejar escalas de trabajo e instrumentos de análisis territorial (Campesino, 1989; Troitiño, 1992). Estos presupuestos, sin embargo, deben demostrarse y no darse por supuesto. Debemos mencionar, igualmente, la existencia de geógrafos que en sus investigaciones «renuncian al territorio», sustituyéndolo por estudios donde dominan las variables economicistas bien como consecuencia de una formación inadecuada, bien, y es lo más frecuente, por deseo de asimilarse a otras disciplinas de mayor consideración social.

La ordenación territorial, objeto de estudio propio del geógrafo, es el elemento de partida de cualquier espacio en el que se desarrollen actuaciones vinculadas al desarrollo local. La escala local es un ámbito de estudio tradicional del geógrafo y ello debería figurar como primera ventaja comparativa en el acercamiento profesional del geógrafo al desarrollo local. Desarrollo local no equivale, como algunos políticos y, desafortunadamente, también algunos técnicos creen a desarrollo municipal, a planteamientos próximos a la denominada como «política de campanario». Esta «teoría política» se basa en la idea de atraer todas las inversiones y beneficios posibles para «mi pueblo» sin pensar en los núcleos próximos, como si los beneficios económicos fueran exclusivamente locales y no existieran las relaciones intermunicipales, de las que se derivan necesariamente complementariedades. De hecho, en la mayoría de los casos el desarrollo local puede y debe ser comarcal, y en algunos, incluso regional, escalas ambas especialmente idóneas para el trabajo de los geógrafos. El ámbito comarcal se adapta notablemente a la comunidad científica de los geógrafos por su capacidad para delimitar los territorios que conforman las comarcas en función de diversas variables integradas, tarea que no resulta muy accesible a otros profesionales que suelen adolecer de comarcalizaciones territoriales más sesgadas. Esa ventaja teórica de la Geografía para delimitar comarcas e incluso regiones tropieza con el problema de que la planificación supramunicipal está poco difundida en España.

Es igualmente necesario hacer referencia a los métodos de trabajo empleados en el análisis territorial. El cotejo de los datos estadísticos con el trabajo de campo, método de trabajo esencial en cualquier estudio geográfico, es necesario para que las cifras estadísticas sean verosímiles. Son numerosos los ejemplos de áreas geográficas catalogadas como deprimidas por la estadística oficial, que distan mucho de serlo si uno se acerca a ellas mediante la observación directa de variables difícilmente cuantificables, pero mucho más creíbles. Resulta necesario cruzar un buen número de variables para conocer satisfactoriamente la situación de partida de un área geográfica, y ello sólo es relativamente factible en contextos geográficos de dimensiones tales que permitan un trabajo de campo exhaustivo, lo que nos remite de nuevo a la escala local, entendida tal y como se definió en párrafos anteriores, como la idónea para el trabajo del geógrafo.

\section{La Geografía y el desarrollo local: potencialidades y carencias de los geógrafos}

Un objetivo fundamental que debe presidir el quehacer geográfico es hacer Geografía, entendida ésta como una ciencia capaz de integrar hechos físicos y humanos con la finalidad 
de comprender, explicar e interpretar la organización del espacio por el hombre. Ese conocimiento debe procurar unos resultados capaces de ser aplicados en beneficio de la sociedad. Uno de los retos más importantes que tiene la Geografía es adquirir prestigio social, que se manifiesta en obtener utilidad social.

El desarrollo local es uno de los campos donde el quehacer del geógrafo puede ser más efectivo ya que, teniendo en cuenta la situación de partida de los territorios en donde se aplican políticas de desarrollo local, es decir, regiones con determinadas deficiencias estructurales, la adopción de políticas que consideren a estos ámbitos de manera integral será fundamental, dada la relevancia concedida a actuaciones basadas en la revalorización de los recursos endógenos, la adopción de prácticas sostenibles social y ambientalmente y la promoción integrada de esos recursos (Gómez, 1995; Esparcia, 2000). Cualquier iniciativa a adoptar deberá considerar las interrelaciones presentes que se establecen entre la estructura socioeconómica (infraestructuras, actividades económicas —agricultura, industria, turismo-, estructura demográfica, etc.) y la estructura ecológica sobre la que éstas se desarrollan, pero también las interconexiones pasadas y la evolución registrada por éstas y que ha dado lugar a la situación presente.

En esa interacción entre medio geográfico y sociedad, el paisaje es una de las cuestiones claves, tanto por su relevancia desde el punto de vista de la ciencia geográfica como de las iniciativas encaminadas a su revalorización y adoptadas por las políticas de desarrollo local (Moltó y Hernández, 2000,b). El profesor Villegas insistía ya a finales de los sesenta sobre las potencialidades de la Geografía como ciencia del paisaje y en la responsabilidad de los geógrafos ante los problemas de su tiempo, rehuyendo la especialización en favor de la visión de conjunto (Campesino, 1989).

Un paisaje rural, entendido como la interacción de una sociedad y un medio, es algo más que una suma de elementos físicos y humanos. Es necesario considerar las interacciones que se han establecido entre ellos, pero no sólo las actuales, sino también las pasadas ya que, en numerosas ocasiones, la estructura actual es resultado de relaciones heredadas y su uso actual se vincula a actuaciones pasadas que pueden ser rentables bien con el aprovechamiento originario, bien con un uso distinto o bien no ser funcionales desde un punto de vista socioeconómico, pero si medioambiental. Ejemplo de ello son los aterrazamientos en áreas de media montaña mediterránea. Su origen se vincula a la roturación de tierras de escasa vocación agrícola coincidiendo con periodos de elevada presión demográfica. Su puesta en valor se llevó a cabo mediante una serie de técnicas que trataban de reducir los posibles procesos de degradación al medio. La aparición de actividades que generaban rentas alternativas a la agricultura supuso su progresivo abandono dadas las dificultades impuestas por el medio para su modernización e intensificación. Esos elementos que en el pasado constituyeron una rémora, son en la actualidad objeto de revalorización con fines turísticos (Calatrava, 1994; Hernández y Moltó, 2000).

En la elaboración de iniciativas encaminadas a paliar los desequilibrios territoriales, como, por ejemplo, un plan estratégico, será necesario conocer los elementos que conforman la estructura natural y la antrópica, más allá del mero elenco de elementos, para identificar las relaciones que se establecen entre ellos. Esta fase resulta fundamental para identificar los rasgos que caracterizan a un determinado territorio y diagnosticar las principales limitaciones, deficiencias y potencialidades con los que cuenta. En este sentido es imprescindible, igualmente, un primer inventario de elementos físicos y humanos susceptibles de convertirse en recursos para el desarrollo o en limitaciones para el mismo. El inventario no debe equivaler a enumeración, no carece de intencionalidad, debe aportar unas 
conclusiones previas sobre la situación existente y acerca de determinadas limitaciones que definan a esa entidad local, supramunicipal, etc.

La Geografía, el geógrafo no siempre, puede ser una ciencia especialmente bien preparada si no para hacer el inventario de todos y cada uno de los elementos, sí para saberlos interpretar en una clave integral y vertebradora de un territorio. El geógrafo no debe aspirar a ser el único especialista capacitado para estudiar con detenimiento todos y cada uno de los elementos de un territorio, pero sí ser el más preparado para identificar e interpretar cómo interactuan entre ellos, una vez le haya sido aportada esa información por profesionales de campos de investigación más específicos. Un ejemplo de representación cartográfica de esos distintos elementos de manera integrada lo constituyen los Sistemas de Información Geográfica (SIG), que nunca deben ser considerados como productos finales de una investigación geográfica aplicada al desarrollo local, sino como herramienta informática de base esencialmente geográfica.

Este diagnóstico es necesario, igualmente, para poder planificar y gestionar las actividades a adoptar en un territorio para paliar las carencias evidenciadas y, sobre todo, desarrollar políticas coherentes que eviten la aplicación de políticas contradictorias que suponen por un lado el despilfarro de recursos y, por otro, generan pérdidas de confianza en la población local. El predominio de un análisis integrado es el principio que define a las actuaciones relacionadas con la planificación y gestión territorial, no obstante, éste en ocasiones presenta un carácter más teórico que real, tal y como corrobora el análisis de algunas de las iniciativas llevadas a cabo en la estructura económica — sectores económicos- y ambiental, que se desarrollarán, sucintamente, en los párrafos sucesivos.

La actividad agraria, al igual que los demás elementos que constituyen la estructura ecológica y la antrópica, puede desempeñar funciones diversas y presentar una potencialidad diferente según el colectivo profesional (agrónomos, economistas, geógrafos, etc.) que lo analice. Frente a una finalidad meramente productiva, los geógrafos deben considerarla como algo más que una actividad económica, como el substrato físico sobre el que se desarrollan una serie de actividades en el medio rural, como, por ejemplo, el turismo. Éste, sin la actividad agraria, aunque algunos consideran que en estos ámbitos donde se aplican políticas de desarrollo rural es una actividad marginal y que podría ser sustituida por completo, perdería su principal recurso y atractivo, ya que ha sido la generadora de esos paisajes que son utilizados por otras actividades (Calatrava, 1996; Hernández y Moltó, 2000).

La agricultura, por tanto, desde un punto de vista de la mera rentabilidad económica directa, no tendría sentido en estos entornos rurales, pero desde un punto de vista territorial, esencialmente geográfico, puede ser fundamental a medio y largo plazo para el mantenimiento de la comunidad y de los valores paisajístico-ambientales. La agricultura, además de una finalidad productiva, presenta una evidente función medioambiental, especialmente en aquellos espacios de escasa vocación agrícola que fueron puestos en cultivo coincidiendo con periodos de fuerte presión demográfica. En ellos, la presencia de una población mínima es indispensable para evitar la génesis de procesos de degradación ambiental por subexplotación. La pluriactividad puede ejercer una notable labor en el mantenimiento de las prácticas agrícolas e indirectamente del medio natural, patrimonial y social. Esta forma de explotación puede ser considerada como no racional desde una óptica estrictamente económica. Sin embargo, en ámbitos rurales donde predominan los secanos extensivos más o menos regresivos, necesitados de grandes extensiones para ser mínimamente rentables, las explotaciones agrarias a título parcial de tipo medio, con un origen histórico diverso, en el que predomina la herencia de la situación y/o de la tierra, mantenidas como complemento 
de rentas, ocio activo y con un fuerte peso de la inercia y el apego a la tierra, son un mal menor o la única solución cara a la conservación del paisaje agrario tradicional y al mantenimiento de ciertas actividades agrícolas (Hernández y Moltó, 2001).

Una reflexión similar se puede efectuar si se analizan los aprovechamientos y las prácticas agrícolas. El cultivo más conveniente desde una óptica agronómica puede no ser el más rentable desde un punto de vista económico y/o social y viceversa. El geógrafo debe intentar aportar una visión integradora, intermedia que no equidistante, entre las distintas posturas, buscando un cultivo que sea admisible desde un punto de vista agronómico, relativamente rentable a un entorno rural donde predomina, por ejemplo, la agricultura a tiempo parcial, pero que sea también conveniente desde una óptica social, cultural y ambiental. Probablemente, el aprovechamiento elegido no será el mejor desde ninguno de los puntos de vista necesariamente sesgados, pero sí podría serlo contando los pros y los contras desde cada uno de los aspectos considerados.

La introducción y difusión de la producción ecológica como una de las líneas de actuación contempladas por las políticas de desarrollo en áreas marginales puede ser favorable en determinados espacios desde un punto de vista agronómico y, por supuesto, ecológico, por la calidad de sus suelos, poco necesitados de insumos, pero puede no serlo desde un punto de vista de la rentabilidad económica y convencimiento social de los protagonistas, porque sean tierras especialmente adaptadas a la agricultura convencional o a la producción integrada. En terrazgos donde los rendimientos son medianamente aceptables, como por ejemplo en la comarca denominada como La Montaña de Alicante ${ }^{1}$, es difícil convencer a los agricultores de que cultiven con prácticas ecológicas porque los potencialmente mejores precios percibidos por sus productos no les compensarían la disminución de rendimientos que necesariamente suele llevar aparejada estas prácticas. No obstante, esta agricultura podría resultar además de conveniente, rentable en tierras en proceso de abandono, con suelos poco profundos y con dificultades de cultivo debido a su escasa aptitud agrológica como sucede en las comarcas interiores de Valencia. En ellas, la productividad, ya escasa con técnicas convencionales, podría no ser mucho menor con prácticas biológicas compensada en este caso además por un aumento de los precios percibidos; cuestión esta fundamental a la hora de conseguir que un agricultor apueste por estos cultivos, tal y como ha acontecido en la cooperativa de Millares (Valencia). La ponderación entre disminución de producción e incremento de la rentabilidad y la existencia de unos mercados estables son otras de las cuestiones que determinan la adopción o no de este tipo de prácticas. La adopción de unas prácticas u otras dependerá, por tanto, de una serie de elementos que deben ser considerados, no sólo de manera aislada sino desde un punto de vista integral por las repercusiones

1 La comarca denominada La Montaña de Alicante «se sitúa en el noreste de la provincia de Alicante, englobando 45 municipios de las comarcas del Alcoià, del Comtat, l'Alacantí y la Marina Alta y la Marina Baja. Espacio que recibe la significativa calificación de «La Montaña». Adjetivo que refleja las características fisiográficas predominantes en esta área supracomarcal. La comarca citada ha sido conocida tradicionalmente por su desarrollo urbano-industrial centrado fundamentalmente en el eje Alcoy-Cocentaina-Muro (Pérez Pérez, 1997), que concentra alrededor del $80 \%$ de la población de este espacio, quedando el resto repartido entre 43 núcleos rurales. La heterogeneidad de este espacio geográfico, marcada esencialmente por la dualidad entre los focos urbano-industriales y los rurales, es aun mayor si tenemos en cuenta que tampoco encontramos ninguna uniformidad dentro de los propios municipios rurales desde el punto de vista territorial, económico y demográfico. Algunos de estos pequeños municipios rurales, como Beniarrés, Benilloba o Alquería de Aznar, gozan de una clara tradición industrial, fruto en buena medida de la influencia del eje anteriormente mencionado, mientras otros siguen teniendo un predominio relativo de la actividad agraria» (Hernández Hernández y Moltó Mantero, 1999, pág. 837) 
territoriales que éstos pueden generar. En esta búsqueda de soluciones que armonicen diversos elementos y no puntos de vista exclusivos, en la visión integrada de un territorio en definitiva, es donde el geógrafo debe mostrar su aportación.

En el campo de una planificación urbana e industrial válida para el desarrollo local son diversos los ejemplos en los que se pone de manifiesto la necesidad de un análisis integral de los elementos que se distribuyen en un territorio y que puede ser fundamentalmente aportada por la Geografía. Un primer ejemplo lo hallamos en un acercamiento exclusivamente economicista a la delimitación de distritos industriales, sin un conocimiento del territorio, sin haber, no ya estudiado, sino, ni siquiera visitado el área geográfica a delimitar. Las cifras estadísticas revelaban, según fórmulas matemáticas, la existencia de tres distritos industriales, uno de mediana entidad (Alcoy) y dos más pequeños (Cocentaina y Muro), en las comarcas de L'Alcoià y el Comtat, según criterios irrebatibles desde el punto de vista de un economista. El conocimiento del territorio de un geógrafo proporcionado por el trabajo de campo demostraría lo absurdo de esta afirmación, ya que esos presuntos tres distritos, que ejercían su influencia sobre unos 40 pequeños núcleos rurales, se incluyen en realidad en un único eje industrial conformado por tres municipios casi conurbados, uno más grande y saturado desde el punto de vista de la disponibilidad de suelo (Alcoy) y otros dos más pequeños (Cocentaina y Muro) con mucho más espacio susceptible de convertirse en industrial, que habían ido recibiendo la deslocalización de la industria e incluso de la población del primero.

Un segundo ejemplo que demuestra la necesidad de una visión integral de los elementos que conforman un territorio se evidencia en la localización y trazado elegido a determinadas infraestructuras viarias, equipamientos o instalaciones industriales, que pueden ser aconsejables desde una coyuntura económica y social, por el beneficio que desde este punto de vista pueden aportar a una comunidad local, pero pueden entrar en conflicto, por ejemplo, con valores ambientales y ser rechazables desde una óptica ecológico-ambiental. En estas situaciones se deberían buscar soluciones geográficas integradas que consideraran todos los elementos implicados y fueran capaces de valorar si el apostar por esas conflictivas ubicaciones va a compensar el daño infringido al entorno, hipotecando su desarrollo a medio y largo plazo.

Estas afirmaciones teóricas se pueden aplicar a numerosos territorios. Podemos continuar analizando, por ejemplo, el caso de estudio anteriormente expuesto, que correspondería a una situación real, pero también prototípica. Supongamos que nos encontramos ante un municipio (Alcoy) con relieve montañoso, de honda tradición industrial, aquejado de un grave problema de deslocalización hacia municipios próximos (Cocentaina, Muro, Ibi, Onil, Castalla, Ontinyent), por falta de suelo industrial en calidad y cantidad. Este proceso de deslocalización industrial lleva aparejado importantes pérdidas demográficas y, sobre todo, una suerte de «depresión colectiva» y de crisis de confianza, incluso superior a lo corroborado por los criterios objetivos, de la casi totalidad de sus habitantes. En ese contexto, la presión social y política de buena parte de la población sobre sus dirigentes políticos llega a ser muy intensa. De este modo, ante la existencia de un emplazamiento idóneo por ser una superficie topográficamente llana (Canal d'Alcoi) y la proximidad a una autovía en construcción, que se presume clave como nudo de comunicaciones (Autovía Central Valenciana A36), los planteamientos estrictamente económicos, políticos y sociales priman por completo a la hora de decidir ubicar allí no ya un polígono industrial más, sino el polígono industrial por excelencia que «todos» reclaman como símbolo de la recuperación socioeconómica del municipio. Frente a ello, «unos pocos», desde posiciones tildadas en 
ocasiones como ecologistas, pero también con planteamientos de racionalidad desde el punto de vista de los recursos, se oponen a esa instalación arguyendo los valores naturales del paraje, su proximidad a un Parque Natural que no cuenta con un PORN y cuya elaboración, actualmente en proyecto, lo incluiría en su totalidad en la zona de pre-parque y, sobre todo, el peligro de contaminación por el hecho de ser la cuenca receptora del principal acuífero abastecedor de agua potable al municipio (el Molinar) y de otros acuíferos que surten a municipios próximos.

Entre ambas posturas pueden existir multitud de posiciones intermedias, pero no existe la equidistancia absoluta. La función de un geógrafo dedicado al desarrollo local, enfrentado a ese dilema, debería ser la de integrar todas las visiones sobre el tema, olvidándose de sí son o no las mayoritarias, y aportar soluciones prácticas y realistas que sean capaces de comprender la presión socioeconómica para promover suelo industrial, sin olvidarse de la grave afectación que puede generarse no sólo en el medioambiente en su conjunto, sino en un recurso natural y económico vital como es el agua potable. Estas soluciones pueden significar, según los casos, desde aceptar el emplazamiento propuesto con todas las limitaciones y garantías de un uso correcto y respetuoso con el medio, hasta proponer la ubicación de polígonos más pequeños en otros lugares menos problemáticos del municipio, pasando, incluso, por la promoción de un polígono mancomunado en un municipio vecino con disponibilidad de suelo abundante y con menos conflictos de uso. La solución que no debe plantearse en ninguna circunstancia es que el técnico encargado de realizar el estudio se limite a describir y plantear los conflictos o a denegar o aceptar sin más el emplazamiento previsto, sin aportar alternativas claras y viables. Es un defecto frecuente entre numerosos geógrafos el limitarse a plantear todas las contradicciones presentes en la resolución de estos problemas sin ir más allá, sin proponer soluciones concretas, prácticas e, incluso, políticamente asumibles. No hemos de olvidar que, en todas estas cuestiones, no ya el geógrafo, sino cualquier técnico en desarrollo local, no es el encargado de ejecutar las acciones sino de proponerlas, pero sí debe saber «vendérselas» al político lo mejor posible para que éste las asuma.

Numerosos son los ejemplos relacionados con la construcción de infraestructuras y, concretamente, el controvertido tema de las comunicaciones. Para desarrollar un territorio cualquiera es necesario dotarlo de una red de comunicaciones mínimamente aceptable, tanto para que lo acerque a los potenciales visitantes o inversores como para que los que residen en él tengan facilidades para salir al exterior en busca de todo aquello que necesiten. En el caso de algunas áreas rurales, se plantea el debate entre aquellos que defienden el aislamiento con el fin de preservar determinados valores culturales y naturales de la «contaminación» urbana exterior y los que argumentan que para que un área rural tenga unas mínimas posibilidades para desarrollarse y superar su marginalidad es necesario que cuente con una inmejorable red de comunicaciones interna y con el exterior de forma que sea plenamente accesible a cualquier coste, tanto económico como de degradación del medio. Cabe plantear, siempre con posiciones integradoras próximas a la Geografía, si no sería posible defender posiciones más o menos intermedias para, según los casos, mejorar en lo posible las comunicaciones, evitando así un aislamiento poco deseable, siempre respetando en lo posible el medio por el que deben discurrir, y, a la vez, restringir el acceso a determinados enclaves valiosos y sensibles a impactos exteriores desde distintos puntos de vista (Hernández y Moltó, 2000).

Otro ejemplo interesante de las opciones que se deben adoptar al plantear el desarrollo sensu estricto de territorios calificados como rurales la encontramos en la revalorización 
de los paisajes asociados a la adopción de políticas de desarrollo rural y en la creciente implantación de aerogeneradores en áreas rurales. No se trata ni de negar sin más en cualquier lugar la instalación de estos aerogeneradores por la contaminación visual y acústica que generan o por la mortandad que ocasionan en determinadas aves, ni de aceptarlos como panacea de desarrollo del municipio que los acepta por los beneficios económicos que a corto plazo le reportan. Conviene planificar a medio-largo plazo, algo muy poco frecuente en general, el modelo de desarrollo elegido para un determinado territorio y evitar posibles contradicciones entre posibles actividades. Cabe preguntarse, por ejemplo, si a un municipio que empieza a contemplar al turismo rural como actividad económica que, complementada con otras, pueda constituirse en factor de desarrollo futuro, le interesa hipotecar ese desarrollo a medio-largo plazo potencialmente perdurable, basado precisamente en el paisaje contra el que pueden atentar los aerogeneradores, a cambio del beneficio rápido pero pasajero proporcionado por estos modernos «molinos». En otros casos, donde el paisaje no es especialmente valioso como factor de desarrollo, puede ser una opción a considerar. En ambos casos, es necesario que se realicen estudios que contemplen todas las variables afectadas, siendo rechazable, la opción contraria, es decir, la toma de decisiones basadas en análisis parciales, que sin embargo, son las más frecuentes. El geógrafo, especialmente dotado para el análisis del paisaje, tiene una oportunidad evidente a la hora de analizar esas cuestiones.

Las actuales políticas dimanadas de las iniciativas comunitarias orientadas a paliar los desequilibrios sociales y territoriales entre diferentes regiones abogan por la adopción de una serie de prácticas que favorezcan un desarrollo sostenible y endógeno. Éstas consideran que la revalorización de los paisajes mediante prácticas asociadas al denominado turismo de interior es una de las posibles actuaciones a potenciar. No obstante, al igual que en la estructura socioeconómica y ecológico-ambiental analizadas sucintamente en párrafos anteriores, se observan notables disparidades según la función que a éste se le asigna desde los diversos campos de conocimiento.

Debemos mencionar, en primer lugar, la confusión existente en el empleo de conceptos como turismo rural, agroturismo, turismo de interior, etc. que en ocasiones son empleados como sinónimos cuando no lo son (Ivars, 2000). El agroturismo, entendido en sentido amplio y superando el concepto clásico, que implica una estancia en una explotación agrícola para colaborar en las tareas agrícolas ${ }^{2}$, podría generar actuaciones más complejas al permitir la interrelación entre diversos sistemas productivos (agricultura, servicios, artesanía, etc.) y ambientales que facilitarían la pluriactividad, si no individual que es compleja, si de la unidad familiar (Moltó y Hernández, 2000,b). Su aceptación implica la adopción de actuaciones integradas y no intervenciones sobre un único sector, que es lo que predomina, en general, en los diferentes ámbitos que se han beneficiado de ayudas europeas, dada la mayor sencillez en su puesta en funcionamiento y la obtención de resultados a corto plazo, que es una opción perseguida por agentes políticos y económicos interesados en obtener resultados rápidos, aún cuando presente ciertas disfuncionalidades (Moltó y Hernández, 2000,a). No obstante, esa actuación integrada en el territorio, más allá de un análisis meramente económico, es la verdadera función del análisis geográfico. Las repercusiones de esas actuaciones en unos ámbitos deprimidos tanto social como territorialmente deben evaluarse

2 «En términos muy generales, el agroturismo es un tipo de turismo rural en el que el componente principal de la oferta turística es la acogida, alojamiento, gastronomía, ocio, participación en tareas, etc., en la explotación agraria» (Calatrava, 2001). 
no sólo desde una óptica meramente cuantitativa, ya que las cifras obtenidas pueden ser poco relevantes si se comparan con otros ámbitos, pero notables desde un punto de vista cualitativo. En esta valoración, el análisis escalar resulta fundamental.

En segundo lugar, es necesario hacer referencia a la revalorización de los recursos naturales. La conciliación entre desarrollo y protección es uno de los objetivos de estas políticas que entre otros principios, deben ser sostenibles. No obstante, en numerosas actuaciones se evidencia, como ya se ha indicado en otros epígrafes, un marcado carácter sectorial al adoptarse actuaciones que, además de hipotecar desarrollos futuros, implican una notable pérdida de calidad paisajística y la adopción de políticas contradictorias como, por ejemplo, el fomento de alojamientos rurales y la instalación de aerogeneradores (Moltó y Hernández, 2000,b). La puesta en valor de ciertos ámbitos se lleva a cabo pensando en los posibles beneficios económicos, convirtiendo esas áreas naturales en «meros jardines» o en otros casos su declaración como áreas protegidas, con vistas a un potencial aprovechamiento turístico dado el atractivo que en la sociedad postproductivista tienen estos espacios. Estas iniciativas, en numerosas ocasiones, se ponen en funcionamiento sin tener en cuenta a las poblaciones locales prohibiéndoles, por ejemplo, ciertas prácticas tradicionales que se consideran poco acordes con un área protegida, lo que genera problemas de rechazo entre esa población por las limitaciones que conlleva y los escasos beneficios que le reportará y potenciando otros usos, que no tienen por el porque ser sostenibles, pero si más adaptadas a posibles visitantes. Afirmaciones estas corroboradas por los análisis realizados a algunos Planes de Ordenación de Recursos Naturales que sancionan ciertos usos ya existentes, aun cuando difícilmente sean compatibles con la declaración de un espacio natural y limitando otros que podrían contribuir a la conservación de esos espacios desde una óptica integral ya que no debemos olvidar que muchos de esos espacios han sido creados por el hombre y por lo tanto son convenientes ciertas intervenciones para conservarlos. Siendo necesario, por tanto, la realización de estudios que contemplen la interacción de los diferentes elementos que intervienen en la estructura ecológica y socioeconómica de esos territorios, identificando potencialidades y limitaciones, como se afirmó en párrafos anteriores.

En tercer lugar, cabe citar la recuperación y/o promoción de determinados elementos patrimoniales y etnológicos, para convertirlos de recursos potencialmente turísticos en productos. Se hace referencia tanto a elementos clásicos, tales como obras artísticas civiles y religiosas de cierta envergadura, como, sobre todo, a otros más novedosos como la arqueología industrial, los yacimientos arqueológicos, los museos etnológicos o los senderos-itinerarios que integran varios de estos potenciales recursos tanto patrimoniales como agroforestales. Desde criterios estrictamente económicos no es rentable, en teoría, invertir en recuperar casi ninguno de estos elementos, si no hay asegurada una mínima rentabilidad final proporcionada por la llegada de turistas. Desde un punto de vista de los especialistas en cada uno de esos elementos (arqueólogos, historiadores, etnólogos, etc.) se afirma, por norma general, que toda obra goza de un interés y un atractivo indiscutible y, como tal, debe conservarse. Cabe plantear nuevamente soluciones intermedias decididas y posibilistas a la vez. No es posible, ni asumible desde un punto de vista económico y de racionalidad funcional recuperar todo, sino únicamente aquellos ejemplos significativos y clarificadores, que requieran inversiones asumibles, accesibles y fáciles de proteger de impactos exteriores. Estos elementos serán simbólicos, no sólo para la atracción de un potencial turismo, sino como hitos de referencia para los habitantes de la confianza en el desarrollo endógeno de su municipio. Igualmente, habrá que tener especial cuidado, por otra parte, en no promocionar 
un potencial recurso como producto turístico consolidado hasta no haberlo afianzado como tal, o el sobredimensionar sus posibilidades, ya que, en ese caso, podemos generar una sensación de «fraude» en el posible visitante, que actuaría de forma contraproducente en el futuro desarrollo turístico de ese municipio. Esta forma de actuar, a pesar de las consecuencias negativas, es muy frecuente; siendo practicada generalmente por intereses esencialmente políticos dada la necesidad de «vender» ciertos resultados a sus potenciales electores, aun cuando éstos sean incipientes y en ocasiones, incluso, irreales.

\section{Conclusiones}

La ciencia geográfica, como afirmaba Orlando Ribeiro en Introdução ao estudo da geografia regional (1962), es una actitud y la manera de trabajar del geógrafo consiste en encontrar un nexo explicativo a los hechos partiendo de su aproximación, observación y ordenación. Tal aseveración nos sitúa ante un conocimiento específico y como tal, precisa de unas determinadas técnicas, pero no por ello más imprecisas o menos valiosas que las utilizadas por otras disciplinas científicas (Olcina Cantos, 1996). La especificidad de la ciencia geográfica vendría determinada por el estudio del territorio y de las dinámicas que se establecen en él como elemento aglutinador. Esta afirmación que podría parecer evidente y obvia si se examinan los planteamientos epistemológicos de esta disciplina, no siempre se cumple en la aplicación práctica de ella. No son extraños los estudios donde el elemento territorial es inexistente; tampoco son inusuales las investigaciones donde no se va más allá del mero inventario de elementos o de un diagnóstico inicial. Es necesario, si se quieren llevar a cabo análisis aplicados, proseguir éstos con la ejecución de las fases relacionadas con la resolución de problemas. Estos estudios deben caracterizarse, igualmente, por un análisis integrado, en el que se ponderen todas las variables. Las soluciones integradoras, la visión integrada del territorio no son una exclusiva de la Geografía, pero los geógrafos deben reivindicar su especial aptitud para proponerlas.

\section{Bibliografía}

AUDIGIER, F. (1992): «La construction de l'espace géographique: propos d'etape sur une recherche en course», Révue de Géographie de Lyon, n 2, pp. 121-130.

AUGÉ, M. (1993): Los no lugares: espacios del anonimato: una antropología de la sobremodernidad, Gedisa, Barcelona, $125 \mathrm{pp}$.

BAILLY, A.S., GUESNIER, B., PAELINCK, J.H.P. y SALLEZ, A. (1987): Comprendre et maîtriser l'espace ou la science régionale et l'aménagément du territoire. Ed. GIPRéclus, 176 pp.

BENKO, G.B. (1990): «Local versus global in social analysis. Some reflexions», en Kuklinski, A.: Globality versus locality, Institute of Space Economy, University of Warsaw, pp. 63-66.

BENKO, G. y STRUHMAYER, V. (eds.) (1997): Space and social theory: interpreting modernity and postmodernity, Blackwell, Oxford, 400 pp.

BOIRA MAIQUES, J.V. (1993): «Percepción del espacio y geografías personales. Una revisión de los estudios realizados en España desde aproximaciones no-analíticas», en Actas del V coloquio de Geografía Cuantitativa, Universidad de Zaragoza, pp. 307-321.

BOISIER, S. (1994): «Postmodernismo territorial y globalización: regiones pivotales y regiones virtuales», en Ciudad y Territorio. Estudios Territoriales, no 102 (2), pp. 597-608. 
BRUNET, R. (1995): «Le terroir. Fin ou renouveau d'une notion», en Cahiers Nantais, $n^{\circ} 45$, pp. 7-12.

CALATRAVA REQUENA, J. (1981): «El uso de espacios abiertos para actividades recreativas como elemento de desarrollo en zonas de montaña: consideraciones sobre los factores que configuran su demanda», en Seminario hispano-francés sobre planificación de recursos en zonas de montaña, Pampaneira (Granada), policopiado.

- (1995): «Actividad agraria y sustentabilidad en el desarrollo rural», en Ramos, E. y Cruz, J. (coords.): Hacia un nuevo sistema rural, MAPA, Madrid, pp. 303-328.

- (1996): «Contingent analysis of the scenic value of sugar cane in the south tropical coast of Granada (Spain): some factors related to willingness to pay», en Azqueta, D. y Pérez, L. (coords.): Gestión de espacios naturales: la demanda de servicios recreativos, McGraw Hill, Madrid.

- (2001): «Agricultura y turismo en el medio rural: aspectos conceptuales y algunas reflexiones sobre su interacción», en Buendía Azorín, J.D. y Colino Sueiras, J. (eds.): Turismo y Medio Ambiente, Ed. Civitas. Estudios y monografías (Economía y Empresa), Madrid.

CAMPESINO FERNÁNDEZ, A.J. (1989): «Geografía y práctica profesional: una asignatura pendiente», en Revista de Geografía, vol. XXIII, Barcelona, pp. 5-30.

CANO GARCÍA, G. (1990): «Escalas y ordenación del territorio desde la Geografía Regional», en Estudios Geográficos, n 199-200, C.S.I.C., Madrid, pp. 339-366.

CARTER, J. y GOLD, J.R. (1993): Space and place: theories of identity and location, Lawrence $\&$ Wishart, Londres.

COX, K.R. (1997): Spaces of globalization: reaserting the power of the local, Guilford Press, Nueva York, 292 pp.

DI MEO, G. 1985): «Les formations socio-spatiales ou la dimension infra-régionale en Géographie», en Annales de Géographie, nº 526, París, pp. 661-689.

- (1991): «La genèse du territoire local: complexite dialectique et espace-temps», en Annales de Géographie, no 559, pp. 273-294.

- (1998): «De l'espace aux territoires: éléments pour une archéologie des concepts fondamentaux de la géographie», en L'Information Géographique, vol. 62, n 3. Ed. Armand Colin, París, pp. 99-110.

DOSI, A. y SCHNELL, F. (1992): Spazio e tempo, Quasar, Roma, 127 pp.

ESPARCIA, J. (1999): «Y llegó la hora de los territorios. Reflexiones en torno al territorio y al desarrollo rural», en Ramos, E. (ed.): El desarrollo rural en la Agenda 2000, MAPA, Madrid.

ESTÉBANEZ ÁLVAREZ, J. (1995): «Globalización, espacio y Geografía», en Polígonos. Revista de Geografía, n ${ }^{\circ}$ 5, Universidad de León, pp. 17-32.

FERRIER, J. (1973): «L'espace, l'aménagement et la Géographie», en L'espace géographique, $\mathrm{n}^{\circ}$ 2, París, pp. 143-156.

GARCÍA BALLESTEROS, A. (2000): «La recuperación del lugar en la era de la globalización», en Lecturas geográficas: homenaje a José Estébanez, Universidad Compluense, Madrid, pp. 1.409-1.416.

GEORGE, P. (1981): Sociedades en mutación, Oikos Tau, Barcelona, 131 pp.

GÓMEZ MENDOZA, J. (1988): «Hacia una estructura general del espacio», en Ería, n 15 , Universidad de Oviedo, pp. 27-39.

GÓMEZ PIÑEIRO, F.J. (1995): «Análisis Geográfico y ordenación del territorio y medio ambiente», en Lurralde. Investigación y Espacio, $\mathrm{n}^{\circ} 18$, Instituto Geográfico Vasco, «Andrés de Urdaneta», Bilbao, pp. 7-20. 
HAMM, B. y JALOWIECKI, B. (1990): The social nature of space, Polish Academy of Science, Committee for Space Economy and Regional Planning, Varsovie, 208 pp.

HARVEY, D. (1989): The conditions of postmodernity: an enquiry into the origins of cultural change, Blackwell, Oxford.

HERNÁNDEZ HERNÁNDEZ, M. y MOLTÓ MANTERO, E. (1999): «Intervenciones en el espacio agrario de la Montaña de Alicante», en El territorio y su imagen, Actas XVI Congreso de Geógrafos Españoles, AGE y Departamento de Geografía de la Universidad de Málaga, pp. 837-846.

- (2000): «El paisaje agroforestal como recurso turístico en la Montaña de Alicante», en Evolución turística de la última década y diseño de futuro, Actas II Jornadas Turismo y Medio Ambiente en las Islas Baleares, Institut d'Estudis Ecologics (INESE), Palma de Mallorca pp. 37-44.

- (2001) «La agricultura: situación actual y perspectivas de un sector tradicional en el marco de las políticas de desarrollo rural», en Revista Española de Estudios Agrosociales y Pesqueros, $\mathrm{n}^{\circ}$ 191, MAPA, Madrid, pp. 9-40.

ISNARD, H. (1978): L'espace géographique, PUF, París, 219 pp.

IVARS BAIDAL, J. (2000): «Turismo y espacios rurales: conceptos, filosofías y realidades», en Investigaciones Geográficas, n 23 , Instituto Universitario de Geografía, Universidad de Alicante, pp. 59-88.

JAMESON, F. (1996): Teoría de la postmodernidad, Trotta, Madrid, 304 pp.

JOHNSTON, R.J., TAYLOR, P.J. y WATTS, M. (eds.) (1995): Geographies of global change: remapping the world in the late twentieth century, Blackwell, Oxford, $462 \mathrm{pp}$.

JORDA BORREL, R. (1995): «Globalización y estrategias regionales en el devenir del siglo XXI», en Cambios regionales a finales del siglo XX, XIV Congreso Nacional de Geografía, AGE y Universidad de Salamanca, pp. 109-111.

KUMAR, K. (1995): From post-industrial to post-modern Society (new theories of the contemporary world), Blackwell, Oxford.

MASSEY, D. y JESS, P. (1995): A place in th world? Places, cultures and globalization, Oxford University Press, 247 pp.

MIRALBES BEDERO, R. e HIGUERAS ARNOL, A. (1993): «Reflexiones sobre el espacio geográfico», en Geographicalia, n 30, Universidad de Zaragoza, pp. 283-294.

MOLTÓ MANTERO, E. y HERNÁNDEZ HERNÁNDEZ, M. (2000, a): «Las políticas de desarrollo rural. Reflexiones sobre sus resultados en la provincia de Alicante», en Los espacios rurales en el cambio de siglo: incertidumbres ante los procesos de globalización y desarrollo, Actas X Coloquio de Geografía Rural, Grupo de Trabajo de Geografía Rural de la Asociación de Geógrafos Españoles y Departamento de Geografía y Sociología de la Universitat de Lleida, pp. 679-685.

- (2000, b): «La integración del turismo, la agricultura y el medio ambiente en las políticas de desarrollo rural en la Montaña de Alicante», en Evolución turística de la última década y diseño de futuro, Actas II Jornadas Turismo y Medio Ambiente en las Islas Baleares., Institut d'Estudis Ecologics (INESE), Palma de Mallorca, pp. 381-388.

NOGUÉ i FONT, J. (1989): «Espacio, lugar, región: hacia una nueva perspectiva geográfica regional», en Boletín de la Asociación de Geógrafos Españoles, no 9, AGE, Madrid, pp. 63-79.

OLCINA CANTOS, J. (1996): «La Geografía hoy: reflexiones sobre el pensamiento geográfico, la región y la docencia de la geografía», en Investigaciones Geográficas, $\mathrm{n}^{\circ} 16$, Instituto Universitario de Geografía, Universidad de Alicante, pp. 93-114. 
PINCHEMEL, Ph. y PIVETEAU, J.L. (1985): «L'espace, concept intégratuer de la géographie», en L'Espace Geographique, $\mathrm{n}^{\circ}$ 2, pp. 5-6.

RAFFESTIN, C. (1984): Territorializzazione, deterritorializzazione, riterritorializzazione, Franco Angeli, Milán.

SACK, R.D. (1987): «El lugar y su relación con los recientes debates interdisciplinarios», en Documents d'Anàlisi Geogràfica, $\mathrm{n}^{\circ}$ 12, Universidad Autónoma de Barcelona, pp. 223-241.

SANTOS, M. (1993): «Los espacios de la globalización», en Anales de Geografía, $n^{\circ} 13$, Universidad Complutense, Madrid, pp. 69-77.

- (1996, a): De la totalidad al lugar, Oikos Tau, Barcelona, 167 pp.

- (1996, b): «Los nuevos mundos de la Geografía», en Anales de Geografía, no 16, Universidad Complutense, Madrid, pp. 15-27.

- (2000): La naturaleza del espacio. Técnica y tiempo. Razón y emoción, Ariel, Barcelona, $348 \mathrm{pp}$.

TORTOSA, J. M (coord.) (1997): De lo global a lo local: relaciones y procesos, Club Universitario, Alicante, $157 \mathrm{pp}$

- (1998): La pobreza capitalista: sociedad, empobrecimiento e intervención, Tecnos, Madrid, $156 \mathrm{pp}$.

TURCO, A. (1988): Verso una teoria geografica della complessità, Unicopli, Milán.

TROITIÑO VINUESA, M.A. (1992): «Dimensión aplicada y utilidad social de la Geografía humana», en Ería, no 27. Universidad de Oviedo, pp. 57-73.

- (2000): «Geografía XXI», en Noticias Geográficas, n 26 , AGE, Madrid, pp. 6-7.

TUATHAIL, G. O. (1998): «Political Geography III: dealing with deterritorialization», en Progress in Human Geography, n 22 (1), pp. 81-93. 